\title{
PRIMARY ANGIITIS OF THE CENTRAL NERVOUS SYSTEM IN PEDIATRIC PATIENTS: CASE REPORT AND LITERATURE REVIEW
}

Daniella Thiemi Ito Sangara ${ }^{1}$, Tatiane Fortuna1,*, Rafaela Zarpelon Kunz ${ }^{1}$, Andrea Natália Montenegro Pérez ${ }^{1}$, Bruno Trevisan¹, Gabriela Sasso Padilha ${ }^{1}$, Maria Lucia Lemos Lopes ${ }^{1}$, Tatiana Freitas Tourinho ${ }^{1}$, Luana Ribeiro Carlos ${ }^{1}$, Maria Odete Esteves Hilario', Lilian Scussel Lonzetti ${ }^{1}$, Thiago Willers ${ }^{1}$, Rafael Coradin ${ }^{1}$

1.Universidade Federal de Ciências da Saúde de Porto Alegre, Porto Alegre (RS), Brazil.

*Corresponding author: tatianefortuna@yahoo.com.br

\section{BACKGROUND}

Primary angiitis of the central nervous system (PACNS) affects two times more men than women, with an average age of 50 years and can occur at any age. In pediatric patients, they affect 12-53/100,000 children under 17 years of age, increasing its incidence as the child ages. Its diagnosis is based on clinical history of neurological findings, cerebral angiography with changes suggestive of vasculitis or biopsy showing vasculitis and the absence of signs of systemic vasculitis. The use of glucocorticoids associated with immunosuppressants improves the prognosis of morbidity and mortality in these patients. The aim of this paper is to report the case of a pediatric patient with PACNS treated at a reference hospital in Rio Grande do Sul.

\section{CASE REPORT}

Female patient, 11 years old, brought to the emergency room by her mother after a report of severe headache associated with left hemiplegia. The patient's mother denies continuous use medications, comorbidities and previous hospitalizations, family history for ischemic and thromboembolic events and menarche. During hospitalization, cranial magnetic resonance angiography was performed showing the presence of stenosis in the right internal carotid artery and hypertensive signal area in T2/FLAIR with correspondence in apparent diffusion coefficient (ADC) map in the caudate, thalamus, insula, prefrontal cortex, postcentral and precentral gyrus. In laboratory tests, patient with no change in the screening for thrombophilia (lupus anticoagulant, anticardiolipin, homocysteine, protein C, protein S, antithrombin III, factor VIII, factor V Leiden and APTT), nonreactive ANA, nonreactive rheumatoid factor and VSG within normal limits. The patient was discharged from hospital with prednisone and cyclophosphamide, without complaints, motor deficits, able to follow up on an outpatient basis.

\section{CONCLUSION}

PACNS in pediatric patients is infrequent and does not have well-established specific diagnostic criteria. Among the symptoms presented, $80 \%$ claim headache, $78 \%$ focal deficit and $62 \%$ hemiparesis. Diagnosis must be established based on clinical history, imaging tests and suggestive absence of other pathologies. Correct diagnosis and treatment increase the prognosis of the case.

\section{KEYWORDS}

Vasculitis, Central nervous system, Child. 